\title{
Espèces nouvellement signalées pour la flore ptéridologique de la République Démocratique du Congo
}

\author{
Jean de Dieu MANGAMBU MOKOSO ${ }^{1,2^{*}}$, van Ruurd DIGGELEN ${ }^{5}$, \\ Jean Claude MWANGA MWANGA ${ }^{3}$, Honorine NTAHOBAVUKA ${ }^{4}$ et \\ Elmar ROBBRECHT ${ }^{5}$ \\ ${ }^{1}$ Université d'Anvers/Belgique, Département de Biologie, Universiteitsplein 1-C, B-2610 Antwerp-Wilrijk \\ Belgium. \\ ${ }^{2}$ Université Officielle de Bukavu, Faculté des Sciences, Département d'Ecologie et Gestion des Ressources \\ Végétales, B.P. 570, Bukavu, RD Congo. \\ ${ }^{3}$ Centre de Recherche en Sciences Naturelles de Lwiro, Département Biologie et Systématique végétale, \\ Direction Scientifique de Bukavu, RD.Congo. \\ ${ }^{4}$ Université de Kisangani, Faculté des Sciences, Département d'Ecologie et Gestion des Ressources Végétales, \\ B.P 2012 Kisangani, R.D Congo. \\ 5 Jardin Botanique National de Belgique, Département des Plantes Vasculaires, Domaine de Bouchout, \\ Nieuwelaan 38, B-1860 Meise, Belgique. \\ *Auteurs correspondant, E-mail : JeandeDieu.MangambuMokoso@student.ua.ac.be, \\ mangambu2000@yahoo.fr
}

\section{RÉSUMÉ}

Nous signalons la présence des espèces nouvellement reportées pour la flore Ptéridologique de la République Démocratique du Congo (RDC), découvertes lors de notre exploration dans la forêt des montagnes du Parc National de Kahuzi-Biega (PNKB), et indiquons leurs conditions écologiques et leurs distributions géographiques. La méthodologie utilisée est celle de traitement taxonomique d'herbier sur les observations macroscopique et microscopique, des analyses chorologique et écologique des spécimens récoltés au PNKB dans la zone des montagnes. Ces recherches ont conduit à signaler 22 espèces nouvelles pour la flore Ptéridologique de la RDC en général et du PNKB en particulier. Parmi ces espèces, la majorité sont Afromontagnardes africaines, certaines sont Plurirégionales africaines, tandis que Pteris repens et Triplophyllum varians montrent une large disjonction. Une espèce, Adiantum incisum, est Paléotropicale. Ces espèces vivent dans des endroits des milieux hydromorphes et humides, les autres sont exposées à la luminosité, tandis que certaines sont épilithes dans l'étage afro-subalpin. Les résultats obtenus montrent que la connaissance de la flore de la RDC reste encore fragmentaire malgré plus d'un siècle de multiples explorations et de récoltes végétales réalisées.

() 2013 International Formulae Group. All rights reserved.

Mots clés: Espèces nouvelles, Pteridophytes, RDC, flore d'Afrique Centrale.

\section{INTRODUCTION}

Le continent africain compte actuellement 1441 espèces de Ptéridophytes regroupées en plus de 135 genres et une cinquantaine de familles (Roux, 2009). La majorité de ces espèces appartiennent à la 
flore de Madagascar et des Mascareignes et de la région afro-montagnarde (Hemp, 2002 ; Aldasoro et al., 2004 ; Autrey et al., 2008 ; Roux, 2009). Plumptre et al. (2003) démontrent que les régions montagnardes de l'Afrique centrale et de l'Est sont constituées en majorité de domaines de conservation et abritent des flores exceptionnelles et riches, avec une singularité qui est le taux d'endémisme élevé (White et Edwards, 2001 ; Basabose et Yamagiwa, 2010), avec plus ou moins 3000 espèces endémiques dénombrées (Plumptre et al., 2003). Ces espèces forment des végétations aussi originales que diverses: forêts montagnardes riches en épiphytes (Hemp, 2002 ; White, 1986, 1993), prairies d'altitude, bambouseraies, buissons d'immortelles où tapis de mousses et de lichens qui se succèdent sur les pentes et forment des tourbes dans les fonds de vallées humides et aux sommets enneigés (White et Vande Weghe, 2008).

La République Démocratique du Congo (RDC) vient en deuxième rang après Madagascar du point de vue diversité de Ptéridophytes. 303 espèces et 81 genres de Ptéridophytes y ont été dénombrés (Roux, 2009). Plusieurs travaux de terrain sur les Ptéridophytes de la RDC ont été réalisés et le matériel récolté a fait l'objet des publications diverses : Bizzarri (1981, 1983); Lawalrée (1990, 1990) ; Fischer (1996) ; Mangambu et Ntahobavuka (2008); Mangambu et al. (2010) ; Pichi Sermolli (1983, 1985) et 16 volumes de Ptéridophytes parus dans la « Flore d'Afrique centrale ».

Dans ces inventaires historiques, les deux monts (Kahuzi et Biega) du Parc National de Kahuzi Biega (PNKB) et quelques marais ont été pris en compte dans les grandes expéditions et récoltes en RDC. Malgré ces quelques publications citées pour les Ptéridophytes de la RDC, l'exploitation botanique de cette flore n'est pas avancée bien qu'elle occupe la deuxième place à l'échelle continentale africaine en les comparants aux travaux basés sur les Spermatophytes. C'est pour cette raison, pendant plus ou moins une décennie, nous avons fait des travaux de terrain dans la zone des montagnes du PNKB, entre 1250-3326 m d'altitude, qui se trouve dans les Systèmes montagnards du KivuRuwenzori. Cette zone est formée de massifs, des chaînes et des monts «Mitumba » dans la région du rift albertin, et se trouve au carrefour des centres d'endémisme GuinéoCongolaise et afro-montagnarde (White, 1986).

L'objectif du présent travail est de signaler la présence des espèces nouvellement reportées pour la flore Ptéridologique congolaise, découvertes lors de notre exploration, d'indiquer leurs conditions écologiques et leurs nouvelles répartitions géographiques. De plus, la présente étude s'intègre aussi dans le vaste chantier entrepris par le Jardin Botanique National de Belgique et le Ministère Congolais de l'Environnement, Conservation de la Nature et Tourisme pour renouveler les études de la connaissance de la flore de la RDC en particulier et de la flore de l'Afrique centrale en générale et aussi à la favorisation sur la connaissance de la biodiversité des montagnes.

\section{MATÉRIEL ET MÉTHODES Milieu d'étude}

Le Parc National de Kahuzi-Biega (Figure 1), est composé de forêts ombrophiles de la plaine et de montagnes (étages submontagnard, montagnard et afro-subalpin ; Mangambu et Ntahobavuka, 2008). Cette végétation s'étalonne entre $600 \mathrm{~m}$ et $3324 \mathrm{~m}$ d'altitude (Basabose et Yamagiwa, 2010). Dans la zone montagnarde règne un climat tropical humide avec deux saisons pluvieuses entre janvier-mai et septembre-décembre, des moyennes des pluies se situant aux environs de 1700-2000 mm/an (Yamagiwa et al., 2005). La température varie selon l'altitude, 
l'humidité atmosphérique est constamment élevée et le sol est superficiel et acide (Mangambu et al., 2010).

\section{Récoltes et identification}

Les recherches actuelles que les auteurs ont mené dans le cadre de cette étude entre avril 2004 et juillet 2011 dans le Parc National de Kahuzi Biega (PNKB), ont permis de récolter 4007 spécimens de Ptéridophytes, soit le double d'herbiers récoltés auparavant par plusieurs chercheurs dans ce Parc dont les lieux où se trouve le matériel examiné et principaux récolteurs sont repris dans le Tableau 1.

La détermination s'est effectuée avec des spécimens de référence de l'herbier du Jardin Botanique National de Belgique (BR) et $\mathrm{du}$ National Herbarium Nederland à Wageningen (WAG). Nos récoltes sont déposées à Herbarium de Lwiro en RDC (LWI), avec des doubles à l'herbier du Jardin Botanique National de Belgique (BR). Pour l'identification, nous nous sommes basés sur des observations macroscopique et microscopique des caractères suivants :

- des caractères qualitatifs (forme de rhizome, présence ou absence d'écailles, couleur des écailles, port général du stipe ou pétiole, type des frondes et nervation, disposition, costa, costules, sores ...),

- des caractères quantitatifs (hauteur de la plante, dimension de fronde, penne, pétiole, rachis ...).

\section{Traitement taxonomique, chorologique et écologique}

La méthodologie utilisée est celle de traitement taxonomique d'herbier. Les phytochories de White (1986, 1993) ont été suivies. Les facteurs écologiques, à savoir le type d'habitat, l'intervalle d'altitude correspondant aux spécimens récoltés et l'adaptation de l'espèce ont été enregistrés sur le terrain. Le nom vernaculaire est cité s'il existe.

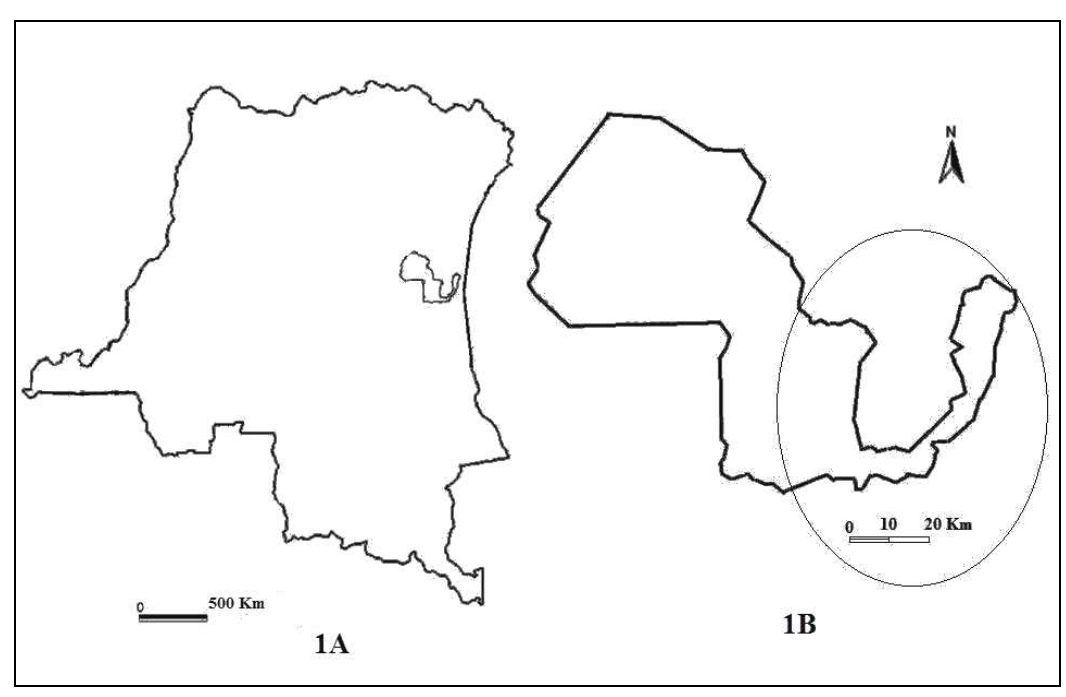

Figure 1: Localisation du Parc National de Kahuzi-Biega (PNKB) et site d'échantillonnage dans le Parc. 1A. Visualisation du PNKB par rapport à la République Démocratique de Congo; 1B. Carte du PNKB (localisé entre $27^{\circ} 33^{\prime}$ 'et $28^{\circ} 46^{\prime}$ de longitude Est et entre $1^{\circ} 36^{\prime}$ et $2^{\circ} 37^{\prime}$ de latitude Sud à l'Ouest du Lac Kivu près de la ville de Bukavu), la zone encerclée représente le site échantillonne. 
Tableau 1 : Localisation du pays et de l'herbier où le matériel a été examiné.

\begin{tabular}{|c|c|c|c|c|}
\hline Pays & Institut & $\begin{array}{l}\text { Acronyme de } \\
\text { l'herbarium }\end{array}$ & $\begin{array}{l}\begin{array}{c}\text { Nombres } \\
\text { des } \\
\text { spécimens }\end{array} \\
\end{array}$ & Récolteurs principaux \\
\hline \multirow[t]{4}{*}{$\mathrm{RDC}$} & $\begin{array}{l}\text { Centre de Recherche } \\
\text { en Sciences } \\
\text { Naturelles de Lwiro }\end{array}$ & LWI & 2490 & $\begin{array}{l}\text { Bashonga, Bequaert, } \\
\text { Christiaensen, J.Petit, Hendrickx } \\
\text { Mangambu, Mwanga mwanga, } \\
\text { Dumbo Ntakiyimana, Troupin et } \\
\text { Van Der Veken }\end{array}$ \\
\hline & $\begin{array}{l}\text { Institut National de } \\
\text { la Recherche } \\
\text { Agronomique } \\
\text { (INERA) Mulungu }\end{array}$ & MLGU & 47 & A. Leonard, Hendrickx et Pierlot \\
\hline & $\begin{array}{l}\text { Institut National de } \\
\text { la Recherche } \\
\text { Agronomique } \\
\text { (INERA) de } \\
\text { Yangambi } \\
\end{array}$ & YBI & 327 & $\begin{array}{l}\text { A. Leonard, Christiaensen, } \\
\text { Demaret, Deuse, Hendrickx } \\
\text { A.Leonard, Pierlot Troupin et } \\
\text { Van Der Veken }\end{array}$ \\
\hline & $\begin{array}{l}\text { Centre de Formation } \\
\text { et de Recherche en } \\
\text { Conservation } \\
\text { Forestière d'Epulu }\end{array}$ & EPU & 496 & Liengola et Mangambu \\
\hline \multirow[t]{4}{*}{ Belgique } & $\begin{array}{l}\text { Jardin Botanique } \\
\text { National de } \\
\text { Belgique (Meise) }\end{array}$ & BR & 2394 & $\begin{array}{l}\text { Bashonga, Christiaensen } \\
\text { Bequaert, Demaret } \\
\text { Deuse; J Petit, Hendrickx, } \\
\text { Mangambu, Ntakiyimana, } \\
\text { Troupin et Van Der Veken }\end{array}$ \\
\hline & Université de Liège & LG & 100 & Troupin et Ntakiyimana \\
\hline & Université de Gent & GENT & 39 & Van Der Veken et Ntakiyimana \\
\hline & $\begin{array}{l}\text { Université Libre de } \\
\text { Bruxelles }\end{array}$ & BRLU & 36 & Masumbuko et Shalukoma \\
\hline Pays-Bas & $\begin{array}{l}\text { Wageningen } \\
\text { Agricultural } \\
\text { University }\end{array}$ & WAG & 58 & Van Der Veken et Christiaensen \\
\hline
\end{tabular}

\section{RÉSULTATS}

Un total de 318 spécimens à frondes fertiles appartenant aux 22 espèces s'est avéré représentatif de taxons non encore trouvés en RDC. Il s'agît surtout de nos propres récoltes (257 collections de Mangambu), mais s'y ajoute bon nombre de récoltes historiques (61 récoltes de Bashonga, Demaret, Christiaensen, Dumbo, Troupin et autres), restées indéterminées ou mal identifiées. La classification adoptée est celle de Roux (2009).

\section{Espèces de Ptéridophytes nouvellement signalées pour la RDC}

Au total, 22 espèces appartenant à 17 genres et 15 familles différents viennent d'être signalées pour la première fois dans la flore ptéridologique de la RDC.

\section{Adiantaceae}

Adiantum incisum Forsk.

Distribution: Angola, Botswana, Burundi, 108 
Madagascar, Malawi, Maurice, Mozambique, Namibie, Nigeria, RDC, Réunion, Rwanda, Seychelles, Somalie, Tanzanie, Togo, Uganda, Zambie, Zanzibar, Zimbabwe et Asie tropical.

Matériel congolais examiné

Etage submontagnard. Mangambu 1725

(LWI) : Kabona, 1580 m.

Etage montagnard. Mangambu 1580

(LWI) : Tshivanga, $2217 \mathrm{~m}$; Mangambu 162

(LWI) : Tshivanga, $2071 \mathrm{~m}$; Mangambu 1810

(LWI) : Mugaba, 2288 m ; Mangambu 2284

(LWI) : Mont. Biega, $2565 \mathrm{~m}$; Mangambu 2286 (BR) : Kasirusiru, Biega $2565 \mathrm{~m}$; Mangambu 2326 (BR, LWI). Tshivanga, 2327 m ; Mangambu 3167 (BR, LWI) : Kasirusiru, 2335 m; Mangambu 3168 (BR, LWI) : Kasirusiru, 2335 m ; Mangambu 3169 (BR, LWI) : Kasirusiru, 2335 m.

Etage afro-subalpin. Mangambu 2279 (LWI) :

Kasirusiru, Biega 2612 m; Mangambu

2312 (LWI) : Mont Kahuzi, 3119 m.

Ecologie et Habitat : Plante terrestre de milieu humide, dans la berge de cours d'eau et sur les collines, exposée au soleil en étage submontagnard. Parfois poussant sur des murs et des grandes roches dans des lieux humides et ombragés. Rarement dans le sous-bois, mais régulièrement sur les bords des routes. Altitude : 1580-3119 m.

\section{Aspleniaceae}

Asplenium abyssinicum Fée

Distribution: Guinée équatoriale (Ile de Bioko), Burundi, Cameroun, Ethiopie, Kenya, Malawi, RDC, Rwanda, Tanzanie et Uganda.

\section{Matériel congolais examiné}

Etage montagnard. Demaret 5023 (BR, YBI) : Kalonge-Madiriri, $2010 \mathrm{~m}$; Demaret 5185b (BR, YBI) : Kalonge-Madiriri, 1700 m; Gupffert 172 (BR, YBI) : Lemera, 1700 m ; Mangambu 2722 (BR, LWI) : Tshivanga, 2196 m; Mangambu 2728 (BR, LWI) : Tshivanga, $2212 \mathrm{~m}$; Mangambu 3061 (BR, LWI): Mugaba, 2344 m Mangambu 3095 (BR, LWI) : Mugaba, 2569 m ; Mangambu 3306 (BR): Tshivanga-Mont Bugulumiza, 2401 m; Dumbo 754 (LWI): Tshibati, 1812 m.
Ecologie et Habitat. Plante de sous-bois dans l'étage montagnard. Hémi-épiphyte sur les arbustes ou épiphyte sur base des grands arbres (souvent sur Chrysophyllum sp. et Hallea sp.) dans les endroits humides ou dans les galeries forestières. Terrestre sur le sommet des collines ou dans des anfractuosités de la roche. Altitude: 1700$2401 \mathrm{~m}$.

\section{Asplenium bugoiense Hieron.}

Distribution. Ethiopie, Kenya, RDC, Rwanda, Tanzanie et Uganda.

\section{Matériel congolais examiné}

Etage submontagnard. Mangambu1472

(LWI) : Ihembe, $1421 \mathrm{~m}$.

Etage montagnard. Mangambu 291 (BR, LWI) : Tshivanga, 2112 m ; Mangambu 1385 (LWI) : Madiriri, 1985 m ; Mangambu 1389 (BR) : Madiriri, $1985 \mathrm{~m}$.

Ecologie et Habitat. Plante épiphyte hygrophile des grands arbres (Ocotea sp. et Lebrunia sp.) des forêts primaires, en général dans l'étage submontagnard. Parfois terrestre ou épiphyte mésophile à l'étage montagnard dans les milieux humides de groupement à Cyathea et/ou rarement dans la forêt primaire. Altitude : 1420 à $2112 \mathrm{~m}$.

Asplenium ceii Pic. Serm.

Distribution. Burundi, Ethiopie, Kenya, Malawi, Mozambique, RDC, Rwanda, Soudan, Tanzanie, Uganda et Zimbabwe.

\section{Matériel congolais examiné}

Etage montagnard. Liengola 1879 (EPU) : Kasirusiru, $2123 \mathrm{~m}$; Liengola 1881 (EPU) : Kasirusiru $2154 \mathrm{~m}$; Mangambu 1356b (BR, LWI) : Madiriri, $1897 \mathrm{~m}$; Mangambu 1358 (BR, LWI) : Madiriri, 1891 m ; Mangambu 1359 (BR, LWI) : Madiriri, 1897 m, Mangambu 3177 (BR, LWI) : Tshivanga, $2154 \mathrm{~m}$; Mangambu 3180 (BR, LWI) : Tshivanga, $2225 \mathrm{~m}$; Mangambu 3183 (BR, LWI) : Tshivanga, $2235 \mathrm{~m}$; Mangambu 3190 (BR, LWI) : Tshivanga, $2296 \mathrm{~m}$; Mangambu 3199 (BR, LWI) : Tshivanga, 2311 m, Dumbo 1012 (LWI) : $1801 \mathrm{~m}$.

Ecologie et Habitat. Plante de l'étage et restreint à l'étage. Epiphyte sciaphile dans des milieux claires ou ouverts sur les sols rocailleux en pente, parfois dans les 
formations arborées pionnières. Rarement dans les galeries forestières et au bord des marais. Altitude : 1890-2315 m.

\section{Asplenium loxoscaphoides Baker}

Distribution. Ethiopie, Kenya, Malawi,

RDC, Tanzanie et Uganda.

Matériel congolais examiné

Etage montagnard. Liengola 1557 (EPU) : Kasirusiru, 2159 m ; Liengola 2255 (EPU) :

Lemera, $1732 \mathrm{~m}$; Mangambu 265 (LWI) : Tshivanga, $2146 \mathrm{~m}$; Mangambu 1564 (BR) : Mugaba, $2212 \mathrm{~m}$

Ecologie et Habitat. Plante hémiépiphyte de base des troncs surtout d'Anthocleista grandiflora Gilg. ou Syzygium rolwandii Sprague et Syzygium cordartum Hochst. ex. Sonder en forêt primaire des sols périodiquement inondés ou milieux marécageux. Rarement rencontrée en forêt secondaire, parfois dans la galerie forestière. Altitude : 1730-2150 m.

\section{Asplenium monanthes $\mathrm{L}$.}

Distribution. Afrique du Sud, Bioko, Burundi, Cameroun, Comores, Ethiopie, Ile Maurice, Kenya, Malawi, Mozambique, Réunion, RDC, Rwanda, Somalie, Soudan, Swanland, Uganda, Tanzanie, Zimbabwe et Zambie.

\section{Matériel congolais examiné}

Etage montagnard. Liengola 561 (EPU) : Mont Bugulumiza, $2143 \mathrm{~m}$; Mangambu 241 (LWI): Tshivanga, $229 \mathrm{~m}$; Mangambu 2358 (BR): Tshivanga, $2115 \mathrm{~m}$; Mangambu 2360 (BR): Tshivanga, 2222 m; Mangambu 2361 (BR, LWI): Tshivanga, 2211 m; Mangambu 2362 (BR): Tshivanga, $2196 \mathrm{~m} ; \quad$ Mangambu 2376 (BR, LWI) :Tshivanga, 2278 m ; Mangambu 2898 (BR, LWI) : Tshibati, $1931 \mathrm{~m}$; Mangambu 2899 (BR) : Tshibati, 1934 m ; Mangambu 2905 (BR) : Tshibati, 2006 m ; Mangambu 3055 (BR, LWI): Mugaba, $2302 \mathrm{~m}$; Mangambu 3056 (BR et LWI) : Mugaba, 2306 m; Mangambu 3058 (BR, LWI) : Mugaba, 2318 m; Mangambu 3071 (BR, LWI) : Mugaba, 2348 m ; Mangambu 3083 (BR, LWI) : Mugaba, $2404 \mathrm{~m}$; Mangambu 3204 (BR, LWI) : Tshivanga, $2309 \mathrm{~m}$; Dumbo705(LWI) : Tshibati, $1809 \mathrm{~m}$.
Etage afro-subalpin. Bashonga 2596 (LWI): Mont Kahuzi, 3187 m; Mangambu 2280 (LWI): Mont Biega $2601 \mathrm{~m}$; Mangambu 297 (LWI): Mont Kahuzi, 3003 m; Mangambu 2309 (LWI): Mont Kahuzi, $3031 \mathrm{~m}$; Mangambu 2313 (LWI): Mont Kahuzi, 3159 $\mathrm{m}$;

Mangambu 2319 (BR, LWI): Mont Kahuzi, $3291 \mathrm{~m}$; Troupin 2292 (BR, GL, LWI, YBI) : Mont Kahuzi, $2951 \mathrm{~m}$.

Ecologie et Habitat. Plante de sous bois, parfois épilithe. Fréquente en forêt hygrophile longeant les bords de ruisseaux dans le tapis muscinal de fourré peu dense. Rarement héliophile dans la végétation de milieux éclairés. Parfois sur les sols rocheux ou calcaires dans les montées des collines à l'étage de montagne. Altitude : 1920-3200 m.

\section{Cyatheaceae \\ Cyathea camerooniana (Hook.) R.M. Tryon var. ugandensis Holttum \\ Distribution. RDC et Uganda \\ Matériel congolais examiné}

Etage submontagnard. Mangambu 1456 (LWI) : Ihembe, 1417 m ; Mangambu 1943 (LWI) : Kamosso, 1567 m.

Etage montagnard. Liengola 1899 (EPU) : Kasirusiru, 2179 m ; Liengola 2094 (EPU) : Tshibati, 1832 m ; Mangambu 1402 (LWI) : Madiriri, 1995 m; Mangambu 1601 (BR) : Tshivanga, $2125 \mathrm{~m}$; Mangambu 1623 (LWI) : Tshivanga, $2091 \mathrm{~m}$; Dumbo 367(LWI): Tshivanga, $2240 \mathrm{~m}$.

Ecologie et Habitat. Plante formant de grands peuplements dans l'étage submontagnard. Elle se rencontre aux bords des rivières, dans les marais et parfois sur la terre ferme. Rarement dans le talus très raide de canal d'amenée d'eau sur l'étage de montagne. Altitude : 1417-2500 m.

\section{Dennstaedtiaceae}

Blotiella hieronymii (Kümmerle) Pic.Serm. Distribution. Burundi, Kenya, RDC,

Tanzanie et Uganda.

\section{Matériel congolais examiné}

Etage submontagnard. Mangambu

1884 (BR,LWI) : Kamosso, $1371 \mathrm{~m}$; 
Mangambu 1917 (LWI) : Kanoiso, 1443 m ; Mangambu 1918 (LWI) : Kanoiso, 1419 m.

Ecologie et Habitat. Espèce rare, rencontrée sur les versants humides de la partie moyenne altitude sous les Uapaca spp. et parfois Ocotea spp. sur le fond des vallées plus au moins marécageuses à Uapaca spp. et Raphia gilletii (De Wild.) Becc. Altitude : 1370-1445 $\mathrm{m}$.

\section{Blotiella stipitata (Alston) Faden}

Distribution. Kenya, RDC et Tanzanie.

Matériel congolais examiné

Etage montagnard. Liengola 1806 (EPU) : Kasirusiru, 2562 m ; Mangambu 2956 (BR, LWI) :

Tshibati, $1921 \mathrm{~m}$; Mangambu 2958 (BR, LWI) : Tshibati, 1918 m ; Mangambu 3078 (BR, LWI) : Mugaba, $2380 \mathrm{~m}$; Mangambu 3079 (BR, LWI): Mugaba, $2388 \mathrm{~m}$; Mangambu 3092 (BR, LWI) : Mugaba, 2342 m; Mangambu 3093 (BR, LWI) : Mugaba, 2339 m; Mangambu 3094 (BR, LWI) : Mugaba, 2334 m; Mangambu 3215 (BR, LWI) : Mulume-munene, $1831 \mathrm{~m}$.

Etage afro-subalpin. Mangambu 2998 (BR, LWI) : Mont Kahuzi, $2861 \mathrm{~m}$; Mangambu 3029 (BR, LWI) : Mont Kahuzi, 3326 m ; Mangambu 3114 (BR, LWI) : Mont Biega, $2623 \mathrm{~m}$.

Ecologie et Habitat. Plante terrestre, rare, fréquente au bord des cours d'eau, dans les endroits humides d'étage montagnard. Parfois sur l'étage afro-subalpin sous les canopées à Podocarpus usambarensis Pilger. Rencontrée aussi au sommet du mont Kahuzi à côté de Helichrysum spp. Altitude: 1830$3326 \mathrm{~m}$.

\section{Dryopteridaceae}

Dryopteris manniana (Hook.) C.Chr.

Distribution. Afrique du Sud, Bioko, Burundi, Côte d'Ivoire, Cameroun, Gabon, Guinée équatorial, Ethiopie, Kenya, Liberia, Madagascar, Malawi, Mozambique, Nigeria, RDC, Rwanda, Sierra Léone, Tanzanie, Uganda et Zimbabwe.

\section{Matériel congolais examiné}

Etage submontagnard. Mangambu 2456

(BR, LWI) : Ihembe, $1527 \mathrm{~m}$.
Etage montagnard. Christiaensen 2781 (BR, GENT, LWI, WAG, YBI) : Madiriri, $1921 \mathrm{~m}$; Liengola 1900 (EPU) : Kasirusiru, $2161 \mathrm{~m}$; Liengola 921 (EPU) : Mugaba, 1892 m ; Liengola 1597 (EPU) : Kasirusiru, 2176 m; Mangambu 1448 (BR, LWI et EPU) : Masanga-Nzila, 2487 m ; Mangambu 3149 (BR, LWI) : Kasirusiru, 2396 m.

Etage afro-subalpin. Mangambu 3021 (BR, LWI) : Mont Kahuzi, 3327 m ; Mangambu 3025 (BR, LWI) : Mont Kahuzi, 3326 m ; Mangambu 3124 (BR, LWI) : Mont Biega, $2664 \mathrm{~m}$.

Ecologie et Habitat. Plante terrestre sub-arborescente de l'étage submontagnard dans les galeries forestières et parfois dans les jachères périodiquement inondées. Plante de sous-bois de forêt primaire en terre ferme ou humide dans l'étage montagnard. Epilithes parfois sur des roches ombreux et humides au sommet des monts Biega et Kahuzi. Altitude : 1527-3326 m.

\section{Elaphoglossaceae \\ Elaphoglossum barteri (Baker) C.Chr}

Distribution. Cameroun, Côte d'Ivoire, Guinée équatorial, Guinée Bissau, Kenya, Liberia, RDC, Sierra Léone, Nigeria, Rwanda et Uganda.

\section{Matériel congolais examiné}

Etage submontagnard. Mangambu 1911 (LWI): Kaniosa, 1478 m; Mangambu 1926 (BR, LWI): Kabuya, 1418 m; Mangambu 1929 (BR, LWI): Kabuya, $1416 \mathrm{~m}$; Mangambu 1957 (LWI): Kamosso, 1556 m; Mangambu 1970 (BR, LWI): Kamosso, 1533 m; Mangambu 1995 (BR, LWI): Mulumemunene, 1667m; Mangambu 001 (LWI): Mulume-munene 1642 m; Mangambu 2016 (LWI): Mulume-munene, $1677 \mathrm{~m}$.

Etage montagnard. Mangambu 1449 (BR, LWI) : Tshivanga, 2487m ; Mangambu 2264 (BR, LWI) : Mont Biega, $2469 \mathrm{~m}$; Mangambu 2841 (BR, LWI) : Madiriri, 1976 $\mathrm{m}$.

Ecologie et Habitat. Hémi-épiphyte des milieux très couverts dans le sous-bois en forêts primaires très humides en étage submontagnard. Dans l'étage montagnard, elle 
est présente dans les galeries forestières humides à Syzygium rolwandii Sprague et Syzygium cordartum Hochst. ex Sonder. ou parfois dans le fond des ravins et dans les marais. Altitude : 1390- $2130 \mathrm{~m}$.

\section{Isoetaceae}

Isoetes welwitschii A. Braun

Distribution. Angola, Botswana, Burundi, Comores, Erythrée, Ethiopie, Ghana, Île Maurice, Kenya, Madagascar, Nigeria, Sud-Soudan, Réunion, Madagascar, RDC, Rwanda, Mozambique et Tanzanie.

Matériel congolais examiné

Etage submontagnard. Mangambu 1881

(BR, LWI) : Lushasha, $1302 \mathrm{~m}$.

Etage afro-subalpin. Mangambu 3116 (BR, LWI) : Mont Biega, $2631 \mathrm{~m}$.

Ecologie et Habitat. Plante très rare, retrouvée dans les sables au bord d'un petit cours d'eau, sur terre humide entre les rochers et sur la latérite dans l'étage montagnard. Retrouvée aussi sur la montée rocheuse ombragée de mont Biega dans l'étage afrosubalpin. Altitude : 1302-2631 m.

\section{Lycopodiaceae}

Huperzia bampsiana Pic.Serm.

Distribution. Burundi, RDC, Rwanda et Tanzanie.

Nom vernaculaire: Tshihungu et Lushalashala (Mashi et Tembo)

\section{Matériel congolais examiné}

Etage montagnard. Christiaensen 2723

(BR, LWI, YBI, WAG) : Mont Kahuzi, 2430 m; Christiaensen 3455 (LWI, WAG, YBI) : Montée de Mont Biega, 2300 m; Mangambu 1572 (BR): Tshivanga, 2210 m; Mangambu 2261 (LWI) : Kasirusiru, montée de Mont Biega, $2459 \mathrm{~m} ;$ Mangambu 2735 (BR, LWI) et 2736 (BR): Tshivanga, $2208 \mathrm{~m}$, Mangambu 3040 (BR, LWI) : Mugaba, 2311 m; Mangambu 3040 (BR, LWI) : Mugaba, 2305 m; Mangambu 3041 (BR): Mugaba, 2298 m, Mangambu 3203 (BR, LWI) : Tshivanga, 2308 m; Mangambu 3207 (BR, LWI) : Tshivanga, $2321 \mathrm{~m}$.
Ecologie et Habitat. Plante épiphyte, hélophile, localisée sur les branches des arbres dans l'étage submontagnard. Parfois hémiépiphytes sur les arbres, dans les milieux rocheux ou sur sols hydromorphes sur Syzygium rolwandii Sprague et Syzygium cordartum Hochst. ex Sonder. ou parfois Macaranga neomildbraediana Lebrun, Myrianthus holstii Engl. et Polyscias fulva (Hiern.) Harms dans les forêts secondaires et dans des forêts de bambous à l'étage montagnard. Altitude : 1420-2470 m.

Polypodiaceae

Pyrrosia schimperiana (Mett. ex Kuhn) Alston var. schimperiana

Distribution. Angola, Cameroun, Cap vert, Côte d'Ivoire, Ethiopie, Kenya, Malawi, RDC, Tanzanie, Zambie, Uganda et Zimbabwe.

\section{Matériel congolais examiné}

Etage submontagnard. Mangambu 2445 (BR, LWI) : Ihembe, $1529 \mathrm{~m}$; Mangambu 2455 (BR, LWI): Ihembe, 1559m; Mangambu 2037 (LWI): Mulume-munene, 1605 m; Mangambu 2458 (BR): 1559 m; Mangambu 2459 (BR, LWI) : 1559 m.

Etage montagnard. Mangambu 72 (BR, LWI) : 2341 met Mangambu 234 (LWI) : Tshivanga, $2229 \mathrm{~m}$.

Etage afro-subalpin. Christiaensen 608

(BR, LWI) : Mont Kahuzi, 2961m ; Mangambu 891 (LWI) : Mugaba, 2606 m; Mangambu 912 (LWI): Mugaba, $2606 \mathrm{~m}$.

Ecologie et Habitat. Plante hémiépiphyte semi-stricte ou préférentielle pouvant coloniser toute la plante hôte dans les forêts secondaires à Hagenia abyssinica (Bruce) J.F.Gmel ou à Macaranga neomildbraediana Lebrun et Croton sp. Parfois seulement hémiépiphyte dans le sous-bois des grands arbres des forêts primaires sur la terre ferme à l'étage montagnard. Par contre, épilithe dans les forêts dégradées de l'étage afro-subalpin. Altitude : 1530-2961 m. 


\section{Pteridaceae}

Pteris repens (C.Chr.) Alston

Distribution. Cameroun, Gabon, Guinée équatorial, RDC et République Populaire du Congo.

\section{Matériel congolais examiné}

Etage submontagnard. Mangambu 1863 (BR) : Lushasha, 1320 m; Mangambu 1892b (LWI) : Kamosso, 322 m; Mangambu 1900 (BR, LWI) : Kamosso, 1322 m; Mangambu 1903b (BR, LWI) : Kaniosa, 1506 m; Mangambu 2024 (LWI) : Mulume-munene, $1667 \mathrm{~m}$; Mangambu 2429 (BR, LWI) : Ihembe, 1517 m; Mangambu 1455 (BR, LWI) : Ihembe, $1417 \mathrm{~m}$; Mangambu 1457a (BR, LWI) : Ihembe, 1411 m et Mangambu 1458 (BR, LWI) : Ihembe, $1416 \mathrm{~m}$.

Ecologie et Habitat. L'espèce est fréquente dans l'étage submontagnard dans les jachères et dans les endroits humides exposés au soleil. Parfois au bord des routes et/ou dans les clairières. Parfois occasionnellement dans les forêts à Ocotea spp. des milieux hydromorphes, périodiquement inondés mais ensoleillés. Altitude : 1320-1670 m.

\section{Sinipteridaceae}

\section{Pellaea angulosa (Bory) Baker}

Distribution. Burundi, Ethiopie, Île Maurice, Kenya, Madagascar, Malawi, Mozambique, RDC, Rwanda, Seychelles, Uganda, Tanzanie et Zimbabwe.

\section{Matériel congolais examiné}

Etage submontagnard. Mangambu 1777

(BR, LWI) : Kabona, 1430 m ; Mangambu 1924 (BR, LWI) : Kaniosa, 1397 m, Mangambu 1925 (BR, LWI) : Kabuya, 1411 m; Mangambu 2021 (LWI) : Mulumemunene, $1559 \mathrm{~m}$.

Etage montagnard. Bashonga 615 (BR, LWI) : Tshibati, $1954 \mathrm{~m}$; Christiaensen 2758 (BR, LWI, WAG, YBI) : Kalonge-Madiriri, 1923 m; Christiaensen 3269 (LWI, YBI) : Kalonge-Madiriri, $1965 \mathrm{~m}$; Liengola 720 (EPU) : Tshivanga, $2253 \mathrm{~m}$; Mangambu 3165 (BR, LWI) : Kasirusiru, 2326 m, Troupin 190 (LG et LWI) : Route Kavumu-Walikale, 1600 m; Troupin 2840 (LG, LWI, BYI) : Route Kavumu-Walikale, $1600 \mathrm{~m}$; Troupin 2899 (LG, LWI, YBI) : Lemera, $1850 \mathrm{~m}$.

Etage afro-subalpin. Troupin 5647 (LG, LWI) : Mont Biega, $2654 \mathrm{~m}$.

Ecologie et Habitat. Plante terrestre des clairières et des bords de cours d'eau dans l'étage submontagnard. Généralement épilithe en forêt humide, le plus souvent dans des endroits plus au moins exposés et dans la vallée des marais en forêt montagnarde. Fréquente dans l'étage afro-subalpin, généralement dans les endroits ouverts et ensoleillés. Altitude : 1390-2655 m.

\section{Tectariaceae \\ Triplophyllum varians (Moore) R.E. Holttum}

Distribution. Angola (enclave de Cabinda), Cameroun, Congo Brazzaville, Côte d'Ivoire Gabon, Guinée équatoriale, Ghana, Liberia, Nigeria, RDC, et Soudan.

\section{Matériel congolais examiné}

Etage submontagnard. Mangambu 1461 (BR, LWI) : Ihembe, $1399 \mathrm{~m}$; Mangambu 1462b (BR, LWI) : Ihembe, 1399m ; Mangambu 1464c (LWI) : Ihembe, $1401 \mathrm{~m}$; Mangambu 1877 (BR, LWI) : Lushasha, 1303 m ; Mangambu 1885 (BR, LWI) : Kamosso, 1363 m ; Mangambu 1991 (BR) : Mulume-munene, $1691 \mathrm{~m}$; Mangambu 1994 (BR) : Mulume-munene, $1672 \mathrm{~m}$; Mangambu 1994b (BR, LWI): Mulumemunene, 1673 m; Mangambu 2008 (LWI) : Mulume-munene, $1622 \mathrm{~m}$; Mangambu 2025a (LWI) : Mulume-munene, $1662 \mathrm{~m}$; Mangambu 2028 (BR, LWI): Mulumemunene, $1632 \mathrm{~m}$; Mangambu 2431 (BR, LWI) : Ihembe, $1511 \mathrm{~m}$.

Ecologie et Habitat. L'espèce est très répandue dans les milieux hydromorphes, aux bords des rivières et les marais et les endroits humides périodiquement inondées dans les forêts hydromorphes à Uapaca guineensis et Anthocleista vogelii. Parfois retrouvée aussi dans les galeries forestières à Macaranga spp. $\mathrm{Au}$ niveau de l'horizon supérieur de l'étage 
submontagnard, elle se rencontre uniquement aux bords des rivières et dans les marais de la forêt primaire à Lebrunia bushaie Staner et parfois à Garcinia punctata Bak et Engl. Altitude : 1300-1675 m.

\section{Thelypteridaceae}

Amauropelta bergiana (Schltdl.) Holttum

Distribution. Cameroun, Comores, RDC, Réunion et Rwanda.

\section{Matériel congolais examiné}

Etage submontagnard. Christiaensen 2724 (BR, LWI, YBI) : Route Kavumu, Walikale, $1515 \mathrm{~m}$ et Troupin 2578 (BR, LG, LWI, YBI) : Route Kavumu-Walikale, 1565 m.

Etage montagnard. Mangambu 2769 (BR, LWI) : Madiriri, $1905 \mathrm{~m}$.

Ecologie et Habitat. Plante terrestre sub-arborescente dans l'étage submontagnard, régulièrement dans les galeries forestières et parfois dans les jachères périodiquement inondées. Rencontrée aussi dans le sous-bois de l'étage de montagne mais rarement épilithe sur des roches ombreux. Altitude: 1500$1905 \mathrm{~m}$.

\section{Metathelypteris vandervekenii Pic.Serm.}

Distribution. Cameroun, Comores, RDC, Réunion et Rwanda.

\section{Matériel congolais examiné}

Etage montagnard. Mangambu 2773b (BR, LWI) : Madiriri, 1905 m et Mangambu 2773c (BR, LWI) : Madiriri, 1907 m ; Dumbo 489 (LWI) : Tshibati, $2069 \mathrm{~m}$.

Ecologie et Habitat. Plante terrestre, rare, uniquement dans le secteur de Madiriri. Elle se rencontre dans les milieux hydromorphes ou périodiquement inondés, parfois au bord des cours d'eau et des zones humides dans le sous-bois. Altitude : 1900$1920 \mathrm{~m}$.

Sphaerostephanos arbuscula (Willd.) Holttum subsp. africanus Holttum

Distribution. Burundi, Ethiopie, Kenya, Madagascar, RDC, Réunion, Tanzanie et Uganda.

\section{Matériel congolais examiné}

Etage montagnard. Liengola 1519 (EPU) : Madiriri, $1951 \mathrm{~m}$; Mangambu 274 (BR, LWI) : Tshivanga, $2287 \mathrm{~m}$; Mangambu 1383 (BR, LWI) : Madiriri, $1985 \mathrm{~m}$; Mangambu 1408 (BR, LWI) : Madiriri, 1951 m ; Mangambu 1408b (BR, LWI) : Madiriri, 1951 m; Mangambu 2789 (BR, LWI) : Madiriri 1897 m; Mangambu 3341 (BR) : Lemera Nord, 1737 m; Mangambu 3351 (BR) : Lemera Nord, 1744 m, Dumbo375 (LWI) : Tshivanga vers Tshibati, $2240 \mathrm{~m}$.

Ecologie et Habitat. Plante de forêt ombrophile de l'étage montagnard. Très fréquente au niveau des zones exposées au bord des routes ou pistes et dans les ouvertures de la forêt, au bord des cours d'eau et dans les zones humides. Parfois rencontrée au bord des cours d'eau ou fond de vallées humides. Altitude : 1000 à 1300 m.

\section{Vittariaceae}

Vittaria volkensii Hieron.

Distribution. Congo, Ethiopie, Kenya, Malawi, Mozambique, RDC, Rwanda, Soudan du Sud, Tanzanie, Zambie et Zimbabwe.

\section{Matériel congolais examiné}

Etage submontagnard. Mangambu 1948

(BR, LWI) : Kamosso, 1543 m.

Etage montagnard. Christiaensen 453 (BR, LWI, WAG, YBI) : Kalonge-Madiriri, 1954 m ; Liengola 980 (EPU) : Mugaba, 2122 $\mathrm{m}$; Liengola 1585 (EPU) : Kasirusiru, 2165 $\mathrm{m}$; Liengola 1938 (EPU) : Tshibati, $1842 \mathrm{~m}$; Liengola 2676 (EPU): Lemera, $1725 \mathrm{~m}$; Mangambu 1551 (LWI) : Tshivanga, $2115 \mathrm{~m}$; Mangambu 1558 (LWI): Tshivanga , 2196 m ; Mangambu 2334 (BR, LWI) : Tshivanga, 2333 m; Mangambu 2369 (BR, LWI) : Tshivanga, $2215 \mathrm{~m}$; Mangambu 2377 (BR, LWI) : Tshivanga, $2270 \mathrm{~m}$; Mangambu 2385 (BR, LWI) : Tshivanga, $2224 \mathrm{~m}$; Mangambu 2386 (LWI) : Tshivanga, 2224 m ; Mangambu 2395 (BR, LWI) : Tshivanga, $2228 \mathrm{~m}$; Mangambu 2881 (BR, LWI): Tshibati, 2010 m ; Mangambu 3161 (BR, LWI) : Kasirusiru, 2478 m; Mangambu 3220 (BR, LWI) : 
Mulume-munene, 2012 m ; Mangambu 3287 (BR) : Tshivanga-Mont Bungulumisa, 2286m; Troupin 1211 (BR, GENT, LG, LWI, YBI) : Mugaba, 2100 m, Troupin 1947 (BR, LG, LWI, YBI) : Mugaba, $2125 \mathrm{~m}$; Troupin 3121 (BR, LG, LWI, YBI) : Marais Musisi, $2100 \mathrm{~m}$.

Etage afro-subalpin. Mangambu 2993 (BR, LWI) : Mont Kahuzi, 2841 m et Mangambu 3127 (BR, LWI) : Mont Biega, $2655 \mathrm{~m}$.

Ecologie et Habitat. Hémi-épiphyte strict ou hémi-épiphyte, rare dans l'étage submontagnard. Fréquente dans l'étage montagnard dans le sous-bois en forêt primaire, rarement dans la forêt à végétation dégradée, ou un peu claire et surtout dans le groupement à Agauria salicifolia (Lam.)Hook. Parfois sur l'étage afrosubalpin sous les canopées à Podocarpus usambarensis Pilger. Altitude : 1543 $2841 \mathrm{~m}$.

\section{Woodsiaceae}

Athyrium scandicinum (Wild.) C.Presl.

Distribution. Burundi, Erythree, Madagascar, Réunion, RDC, Rwanda, SudSoudan, Tanzanie et Zimbabwe.

\section{Matériel congolais examiné}

Etage montagnard. Bashonga 701

(LWI) : Mont Biega, $2541 \mathrm{~m}$; Christiaensen 1481 (BR, LWI, YBI) : Tshivanga, $2090 \mathrm{~m}$; Mangambu 629 (BR, LWI) : Mugaba, $2405 \mathrm{~m}$; Mangambu 2253 (BR): Montée de Mont Biega, 2350 m et Mangambu 2335 (BR, LWI) : Tshivanga, $2335 \mathrm{~m}$.

Etage afro-subalpin. Mangambu 2277 (LWI) : Mont-Biega, $2606 \mathrm{~m}$.

Ecologie et Habitat. Espèce des sousbois humides, des bords de cours d'eau, plus rarement de la végétation éricoïde d'altitude en étage montagnard. Rarement dans l'étage afro-subalpin sous la canopée à Podocarpus usambarensis Pilger. Altitude : 2080-2610 m.

\section{Spectre chorologique et chorotypes}

Les 22 espèces signalées pour la première fois dans la flore ptéridologique de la RDC se distribuent de la manière suivante :

\section{Espèces afromontagnardes}

Douze espèces sont de la région afromontagnarde (Tableau 2). Certaines (Blotiella hieronymi Kümm, Cyathea camerooniana Hook. R.M. Tryon var. ugandensis Holttum, Huperzia bampsiana Pic.Serm. et Metathelypteris vandervekenii Pic.Serm.) sont endémiques du système montagnard Kivu-Ruwenzori dans lequel le PNKB est situé (Figure 2). Trois autres espèces (Asplenium bugoiense Hieron, Asplenium loxoscaphoides Baker et Blotiella stipitata (Alston.) Faden (Figure 3)) sont communes pour quatre systèmes du rift albertin (de l'Ethiopien jusqu'au Uluguru). Cinq espèces (Asplenium abyssinicum Fée, Asplenium ceii Pic.Serm., Pellaea angulosa(Bory) Baker, Amauropelta bergiana (Schltdl.) Holttum et Sphaerostephanos arbuscula (Willd.) Holttum subsp. africanus Holttum) sont des espèces de liaison qui se rencontrent dans la majorité des systèmes montagnards d'Afrique, à savoir le système du Mont Cameroun et les cinq systèmes au Nord du rift albertin (les systèmes Ethiopien, des Imatongs-Usambara, du Kivu-Ruwenzori, des Uluguru-Mlanje et du Chimanimani) ainsi qu'au Madagascar et dans l'archipel des Comores (Figure 4).

\section{Espèces plurirégionales africaines}

Dans ce lot d'espèces signalées pour la première fois en RDC, 9 se trouvent dans plusieurs régions africaines sub-sahariennes. Dans ce groupe, 7 espèces sont plurirégionales africaines, dispersées dans plusieurs régions africaines. Parmi ces plantes, une seule espèce, Dryopteris manniana (Hook) C. Chr., est distribuée de l'Afrique tropicale jusqu'au Madagascar. Une autre, Elaphoglossum barteri (Baker) C.Chr. est distribuée en Afrique de l'Ouest tropicale, pénétrant dans le système montagnard de l'Afrique de l'Ouest 
en passant par le système Kivu-Ruwenzori jusqu'aux forêts de Miombo en Afrique Australe. Trois autres (Athyrium scandicinum(Wild.) C.Presl., Asplenium monanthes L., et Vittaria volkensii Hieron.) sont afro-tropicales; elles se rencontrent dans le centre d'endémisme afromontagnard et pénètrent dans les centres guinéo-congolais, soudaniens et zambéziens. Deux autres, Isoetes welwitschii A. Braun et Pyrrosia schimperiana (Mett. ex Kuhn) Alston var. schimperiana sont Soudano-Zambéziennes et existent dans la mosaïque régionale du Lac Victoria; elles existent aussi dans l'afromontagnard et l'afro-alpin.

Signalons que deux espèces (Pteris repens (C.Chr.) Alston et Triplophyllum varians (Moore) R.E. Holttum) montrent une large disjonction (Figure 5). Triplophyllum varians (Moore) R.E. Holttum est surtout signalée dans les sous-centres Haut et BasGuinéen du centre Guinéo-Congolais. Pteris repens (C.Chr.) Alston se rencontre dans le sous-centre Bas-Guinéen et de quelques endroits isolés dans l'est du centre soudanien. Nous avons retrouvé ces deux espèces dans les montagnes du Kivu, c'est-à-dire dans l'extrémité Est du sous-centre Congolais, séparé par plus ou moins 5000 km des populations bas-guinéennes.

\section{Paléotropicales}

Une espèce (Adiantum incisum Forsk.) a une distribution qui déborde le continent africain, elle se rencontre dans les montagnes d'Afrique et d'Asie tropicales.

Tableau 2: Chorologie des espèces afromontagnardes endémiques nouvellement trouvées en République Démocratique du Congo.

\begin{tabular}{|c|c|c|c|c|c|c|c|}
\hline Espèces & WAfr* & Eth & Im-Us & Ki-Ru & Ul-Ml & Chim & Mad \\
\hline Amauropelta bergiana & + & + & + & + & + & + & + \\
\hline Asplenium abyssinicum & + & + & + & + & + & + & + \\
\hline Asplenium ceii & + & + & + & + & + & + & + \\
\hline Pellaea angulosa & + & + & + & + & + & + & + \\
\hline $\begin{array}{l}\text { Sphaerostephanos arbuscula } \\
\text { subsp. africanus }\end{array}$ & + & + & + & + & + & + & + \\
\hline Asplenium bugoiense & & + & + & + & + & & \\
\hline Asplenium loxoscaphoides & & + & + & + & + & & \\
\hline Blotiella stipitata & & + & + & + & + & & \\
\hline Blotiella hieronymii & & & & + & & & \\
\hline $\begin{array}{l}\text { Cyathea camerooniana var. } \\
\text { ugandensis }\end{array}$ & & & & + & & & \\
\hline Huperzia bampsiana & & & & + & & & \\
\hline Metathelypteris vandervekenii & & & & + & & & \\
\hline
\end{tabular}

Systèmes montagnard suivant White (1983) : systèmes ouest Africain, Ethiopien (Eth), des Imatongs-Usambara, du KivuRuwenzori (WAfr), des Uluguru-Mlanje (Ul-Ml), du Chimanimani (Chim) et Madagascar (Mad). * Les espèces dites sont limitées dans l'ouest-africain au sous-système du Mont Cameroun. 


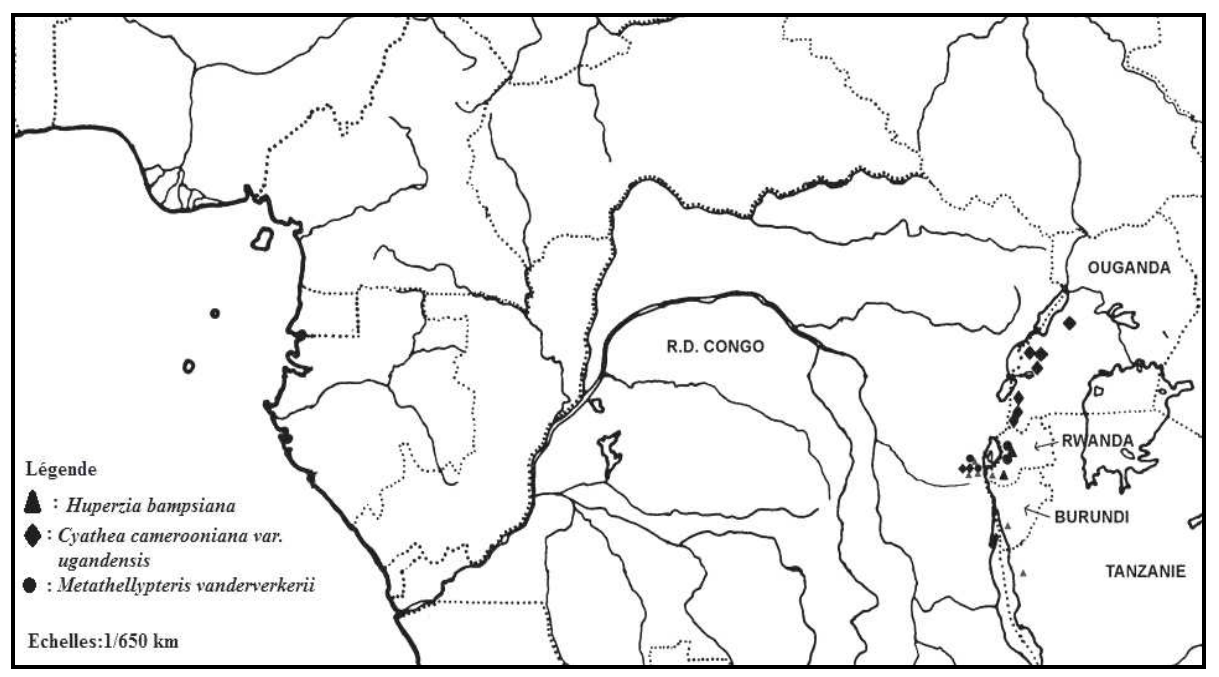

Figure 2: Exemple des espèces du système Kivu-Ruwenzori (chaque symbole utilisé indique les lieux de récolte des espèces. La carte est obtenue par la projection des coordonnées géographiques [latitude et longitude] indiquées sur les étiquettes des herbiers).

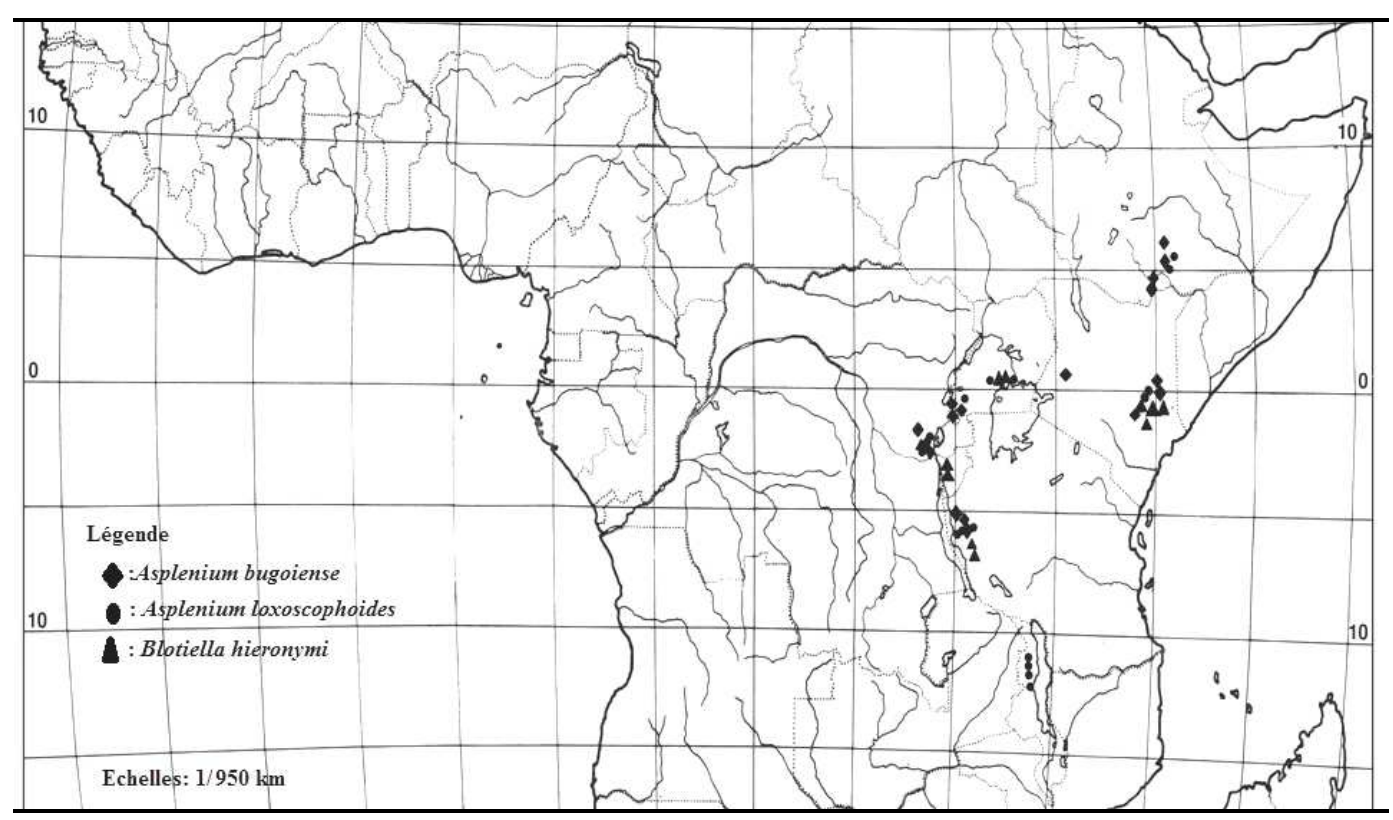

Figure 3: Exemples des espèces répandues dans les quatre systèmes du rift albertin (ces systèmes montagnards sont les suivants : Ethiopien, Imatongs-Usambara, Kivu-Ruwenzori, Uluguru-Mlanje et Chimanimani). 


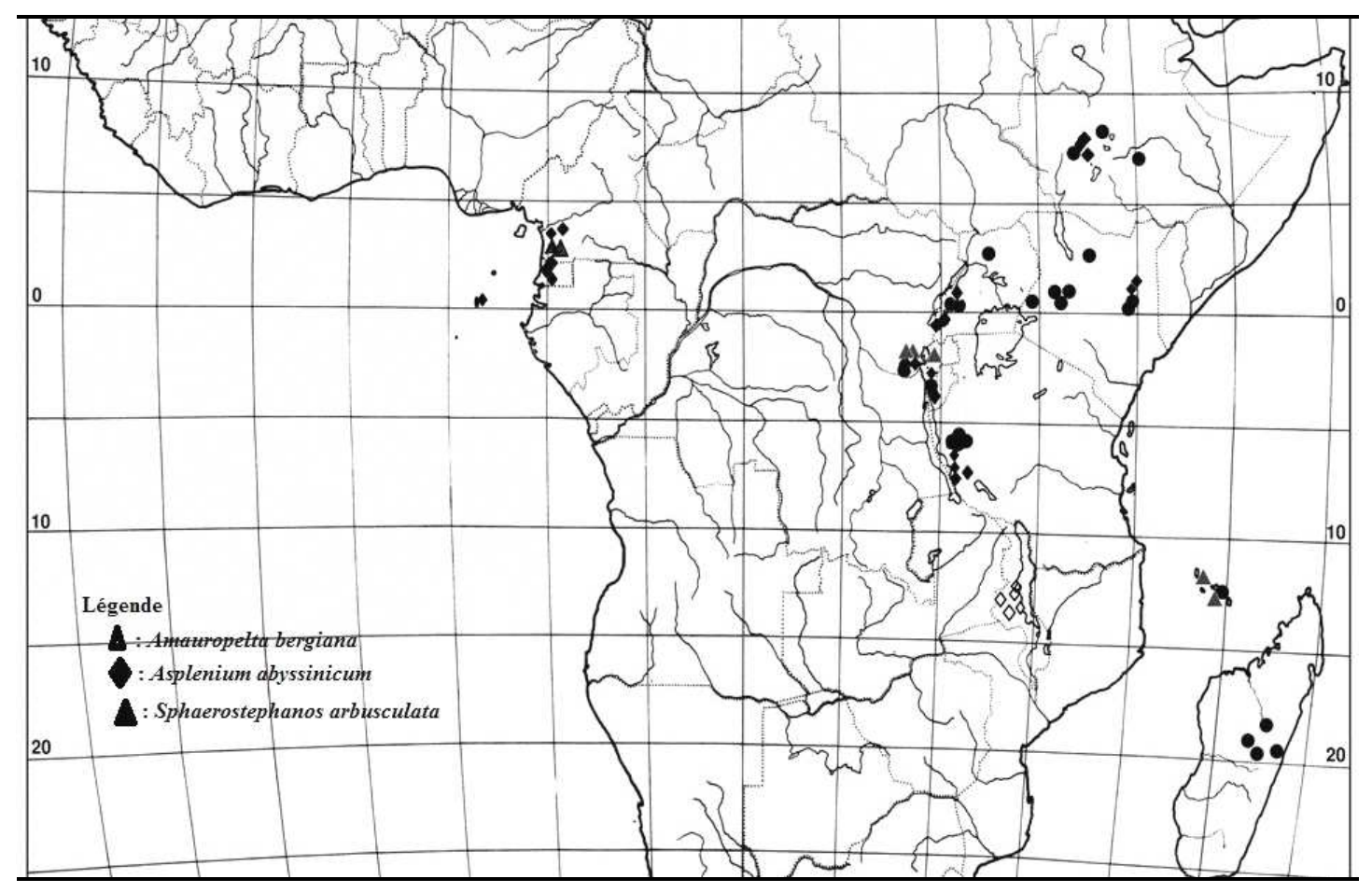

Figure 4: Exemples des espèces de liaisons afromontagnardes (Espèces distribuées uniquement dans le système montagnard africain).

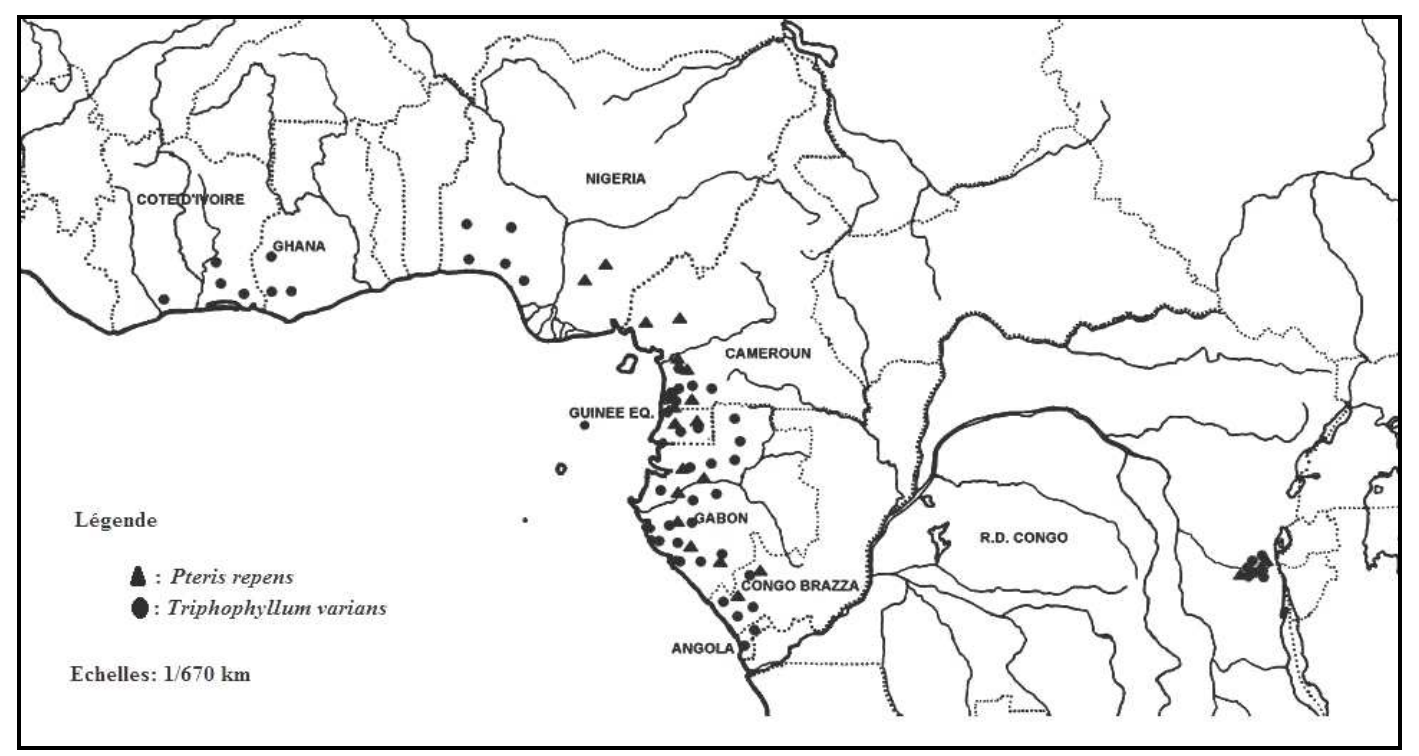

Figure 4: Deux espèces à large disjonction (Pteris repens et Triplophyllum varians, deux espèces jusqu'à présent connues seulement de l'Afrique de l'Ouest, sont signalées pour la première fois dans la flore ptéridologique de l'Est de la République Démocratique du Congo (RD Congo), au Parc National du Kahuzi-Biega). 


\section{DISCUSSION}

Les grandes explorations botaniques en RDC datent de l'époque coloniale, mais les récoltes ne se sont pas faites d'une façon homogène pour toutes les régions. Après l'indépendance en 1960, les inventaires botaniques se sont poursuivis à l'Est de la RDC, au Rwanda et au Burundi (Pichi Sermolli, 1983; Lawalrée 1990, 1991) en 1972 par les chercheurs Belges, Italiens, Polonais et les homologues de l'Afrique centrale. outre ces inventaires de grande envergures, différentes récoltes et inventaires ont été effectués à l'Université de Kisangani et de Kinshasa dans les différentes Réserves environnantes, au Centre de Formation Forestière de la Réserve de Faunes à Okapi (Epulu), au PNKB et la Réserve Naturelle d'Itombwe par les Institutions Universitaires de Bukavu, les ONGs de conservation (Wildlife Conservation Society et World Wildlife Fund for Nature) et au Centre de Recherche en Sciences Naturelles de Lwiro. Dans la majorité des cas, ces travaux avaient des buts écologiques et non taxonomiques. Ainsi, beaucoup d'espèces de la RDC restent encore inconnues, surtout dans sa partie Est qui est montagneuse.

Suivant certains auteurs (Borsdorf et Braun, 2008; Delnatte, 2010), les régions montagnardes sont riches en diversité végétale mais avec une systématique toujours fragmentaire et difficile, suite à des multiples changements que prennent les plantes à différentes altitudes (Kapos et al., 2000 ; Kessler, 2001). Ce sont des zones plus riches en espèces de plantes que les régions de plaines. Les forêts tropicales de montagne abritent les plus riches communautés de plantes de la planète (White, 1996, 1993 ; Barthlott et al., 1996).

En Afrique centrale (RDC, Rwanda et Burundi) en général et en RDC en particulier, plusieurs travaux des récoltes ont contribué à la connaissance de la flore ptéridologique et à l'apparition de 16 volumes des fascicules et une dizaines d'articles (Bizzarri, 1981, 1983 ; Pichi Sermolli, 1983 et 1985; Lawalrée, 1990, 1991; Fischer, 1996; Mangambu et
Ntahobavuka, 2008 ; Mangambu et al., 2010). Par exemple, les inventaires menés en 1972, ont permis à Pichi Sermolli $(1983,1985)$ de découvrir 9 nouvelles espèces de Ptéridophytes, Aleuritopteris vandervekenii Pic.Serm. (RDC), Pteris auquieri Pic.Serm. (Rwanda, Burundi), P. microlepis Pic.Serm (Rwanda), Vandenboschia inopinata Pic.Serm (RDC, Rwanda, Burundi et divers pays d'Afrique tropicale, Madagascar et Mascareignes), Blotiella bouxiniana Pic.Serm (Rwanda, Burundi), B. trichosora Pic.Serm (Burundi), Macrothelypteris rammelooi Pic.Serm (Rwanda), Metathelypteris vandervekenii Pic.Serm. (Rwanda) et Christella burundensis Pic.Serm. (Burundi). Notons aussi qu'il avait signalé 23 espèces pour cette partie de l'Afrique centrale. Les mêmes inventaires ont permis à Bizzarri (1981) d'identifier deux espèces du genre Selaginella : S. vanderystii Bizzarri (rencontré dans le bassin du Congo en RDC), et Selaginella kivuensis Bizzarri dans les montagnes de Kivu, l'Est de la RDC.

Actuellement, l'espèce Selaginella kivuensis Bizzarri n'est pas connue uniquement à l'Est de la RDC; elle se retrouve aussi dans le bassin du Congo (Réserve Naturelle de Lomako et aux environs de Bikoro en RDC tout proche du Parc National de Salonga Nord) et forme une large disjonction jusqu'au pic de le Pico Basilé, plus haute montagne de l'île de Bioko en Guinée équatoriale (Bizzarri, 1985).

Les spécimens provenant du Kivu en RDC et de l'escarpement du Rwanda ont amenés Lawalrée (1990) à la découverte de 2 espèces nouvelles d'Arthropteris de la famille de Nephrolepidaceae (Arthropteris anniana Lawalrée et Arthropteris antungupffertiae Lawalrée) pour l'Afrique. A partir de ce matériel récolté dans la partie basse altitude du PNKB, Lawalrée (1991) a décrit une nouvelle espèce (Arthropteris charletiana Lawalrée) en RDC.

En ce qui concerne le cas de large disjonction remarquable observée (Pteris repens (C.Chr.) Alston et Triplophyllum varians(Moore) R.E. Holttum), elle s'ajoute à 
une longue série d'observations de disjonctions similaires rapportées et souvent expliquées par la théorie des refuges glaciaires. Cette hypothèse admet que durant les périodes glaciaires du quaternaire, la forêt d'Afrique centrale a fortement régressé pour occuper de petites poches appelées refuges forestiers (Sosef, 1994 ; Robbrecht, 1996 et Stévart, 2003).

Deux types de refuge sont généralement reconnus : ceux de plaine et ceux de montagne. Tant par leur écologie que par leur aire de distribution, ces espèces peuvent être considérées comme des marqueurs pour les refuges de montagne. En guise d'exemple, on peut citer les cas des espèces Impatiens burtonii Hook.f et Impatiens mannii Hook.f qui sont très répandues dans le système montagnard de l'Afrique de l'ouest (montagnes du Cameroun, de Bioko et du Gabon) et se retrouvent dans le Kivu-Ruwenzori (Grey, 1980). La seconde se prolonge jusqu'au niveau du système montagnard d'UluguruMlanje. Lachenaud et Jongkind (2010) démontrent que Chassalia pteropetala (K.Schum.) Cheek est très répandue dans le Kivu-Ruwenzori, elle est ponctuellement présente dans la zone de transition SoudanoCongolais et est observée dans les montagnes Ouest-africaines. Elaphoglossum kivuense Schelpe connue avant comme endémique du Kivu-Ruwenzori est actuellement aussi rapportée des montagnes des systèmes Ethiopien et des Imatongs-Usambara ainsi que dans la Zone de transition Guinéo-Congolaise/ Soudanienne (Roux, 2009).

Les résultats obtenus confirment l'hypothèse d'Aldasoro et al.(2004), qui annonce que les aires de distributions des Ptéridophytes sont très variables: les unes couvrent de grandes étendues, les autres se réduisent à des petits endroits. De cette manière, il y a des plantes endémiques, plurirégionales, couvrant de grandes superficies et des plantes néotropicales, paléotropicales, sub-cosmopolites et cosmopolites (Aldasoro et al., 2004 ; Autrey, 2008 ; Roux , 2009 ).
Nous venons de constater que l'examen d'un vaste échantillonnage de plantes récoltées localement et appartenant à un groupe taxonomique cible dans lequel on se spécialise a permis de signaler de nouvelles espèces et d'étaler des détails descriptifs de certaines Ptéridophytes.

D'autre part, nos prédécesseurs avaient à leur disposition qu'un faible nombre d'échantillons par rapport à nos récoltes d'une décennie dans notre zone d'étude. De plus nous relevons aussi que plusieurs parties de la RDC ne sont pas encore prospectées par les scientifiques, la majorité des Parcs Nationaux et Réserves Naturelles étant créés après l'indépendance (Anonyme, 2010). Donc, les études de terrain réalisées viennent de ramener le nombre des Ptéridophytes (Fougères et leurs alliés) de 81 à 85 genres (nouveaux genres: Amauropelta, Isoetes, Metathelypteris, Sphaerostephanos). Les mêmes résultats montrent que la connaissance de la flore de la RDC reste encore fragmentaire malgré plus d'un siècle de multiples explorations et de récoltes végétales réalisées. Ainsi, nous suggérons que des chercheurs locaux soient soutenus financièrement, pour réaliser de multiples récoltes de plantes en général et de Ptéridophytes en particulier.

\section{REMERCIEMENTS}

Le présent travail s'est réalisé grâce à l'appui matériel et financier accordé par la Coopération Technique Belge (CTB). Nous remercions aussi l'UNESCO à travers le gouvernement flamand (Belgique) pour leur contribution aux travaux de terrains et des voyages dans les herbariums pour la vérification et observation du matériel de référence.

\section{REFERENCES}

Agnew A, Agnew S. 1994. Upland Kenya Wild Flowers, East African Natural (2nd edn). East Africa Natural History Society: Nairobi, Kenya.

Aldasoro JJ, Cabezas F, Aedo C. 2004. Diversity and distribution of ferns in sub- 
Saharan Africa, Madagascar and some islands of the South Atlantic. Journal of Biogeography, 31: 1579-1604.

Anonyme. 2010. Quel avenir pour les forêts de la République démocratique du Congo ? Instruments et mécanismes innovants pour une gestion durable des forêts. Bruxelles, Rue Haute, Belgique.

Autrey J, Bosser J, Ferguson I. 2008. Flore des Mascareignes - Ptéridophytes Psilotacées à Marsiléacées. IRD: Paris.

Barthlott W, Porembski S, Szarzynski J, Mund JP. 1996. Phytogeography and vegetation of Tropical inselbergs. In Phytogéographie Tropicale Réalités et Perspectives, Guillaumet J-L, Belin M, Puig (eds). Phytogéographie Tropicale : Colloque International en Hommage au Professeur Schnell: Paris; 15-24.

Basabose AK, Yamagiwa J. 2010. Four decades of research on primates in Kahuzi-Biega. Gorilla Journal, 38: 3-6.

Bizzarri MP. 1985. Flore d'Afrique centrale (Zaïre, Rwanda, Burundi). PtéridophytesSelaginellaceae. Meise/Belgique, 55p.

Bizzarri MP. 1981. Two New Species of Selaginella (Selaginellaceae) from Zaire. Bulletin du Jardin Botanique National de Belgique, 51(1/2), 219-224

Bizzarri MP. 1983. Two New Species of Selaginella (Selaginellaceae) from Zaire. Bulletin du Jardin Botanique National de Belgique, 53(1/2): 171-176.

Bizzarri MP. 1985. Flore d'Afrique Centrale (Zaïre, Rwanda, Burundi). PtéridophytesSelaginellaceae. Jardin Botanique National de Belgique : Bruxelles ; 55 p.

Borsdorf A, Braun V. 2008. Panorama de la recherche sur la montagne en Europe et dans le monde. Revue de géographie Alpine, 96(4): 101-116.

Delnatte C. 2010. Le gradient altitudinal sur les sommets tabulaires de Guyane Basé sur l'étude des Arecaceae, des Melastomataceae et des Ptéridophytes. Thèse de doctorat, Université des Antilles et de Guyane, p. 292.

Fischer E. 1996. Die Vegetation des Parcs National de Kahuzi-Biéga, Sud-Kivu,
Zaïre. Franz Steiner Verlag: Stuttgart; $239 \mathrm{p}$.

Gentry AH. 1992. Tropical forest biodiversity: distributional patterns and their conservational significance. Oikos, 63 : 19-28.

Grey W. 1980. Impatiens of Africa. Balkema/Rotterdam.

Hemp A. 2002. Ecology of the pteridophytes on the southern slopes of Mt. Kilimanjaro. Part I: Altitudinal distribution. Plant Ecology, 159: 211239.

John R. 1991. Pteridophytes of Tropical East Africa: A Preliminairy Chech-List of the Species. Royal Botanic Gardens: Kew.

Kapos V, Manica A, Aveling R, Bubb P, Carey P, Entwistle A, Hopkins J, Mulliken T, Safford R, Stattersfield A, Walpole MJ, Balmford A. 2010. Defining and measuring success in conservation. In Trade-offs in Conservation: Deciding what to Save, Leader-Williams N, Adams WM, Smith RJ (eds). Wiley-Blackwell Publishing Ltd: Oxford, UK; 73-93.

Kessler M. 2001. Patterns of diversity and range size of selected plant groups along an elevational transect in the Bolivian Andes. Biodiversity and Conservation, 10: $1897-1921$.

Lachenaud O, Jongkind C. 2010. Three new or little-known Chassalia (Rubiaceae) species from West and Central Africa. Nordic Journal of Botany, 28: 13-20.

Lawalrée A. 1991. Arthropteris charletiana (Nephrolepidaceae), espèce nouvelle du Kivu (Zaïre). Bulletin $d u$ Jardin Botanique National de Belgique, 61(2): 356-357.

Lawalrée A. 1990. Deux espèces africaines nouvelles d'Arthropteris (Nephrolepidaceae). Bulletin du Jardin Botanique National de Belgique, 60(2): 317-324.

Mangambu JD, Habiyaremye M, Lina A, Ntahobavuka H. 2010. L'importance du groupement à Cyathea manniana dans la biodiversité du Parc National de Kahuzi - 
Biega, R. D. Congo. Geo-Eco-Trop., 34: 45-63.

Mangambu M, Ntahobavuka H. 2008. Flore analytique des Ptéridophytes de KahuziBiega, R.D Congo: I Cyatheaceae. Annales des Sciences de l'Université Officielle de Bukavu, 1(1): 51-53.

Pichi S. 1983. A contribution to the knowledge of the Pteridophyta of Rwanda, Burundi, and Kivu (Zaire) I. Bulletin du Jardin Botanique National de Belgique, 53(1/2): 177-284.

Pichi S. 1985. A contribution to the knowledge of the Pteridophyta of Rwanda, Burundi, and Kivu (Zaïre) II. Bulletin du Jardin Botanique National de Belgique, 55(1/2): 123-206.

Plumptre AJ, Behangana M, Ndomba E, Davenport T, Kahindo C, Kityo R, Nkuutu Ssegawa P, Eilu G, Owiunji I. 2003. The Biodiversity of the Albertine Rift. Albertine Rift Technical Reports $\left(N^{\circ} 3\right)$. Unpublished report, Wildlife Conservation Society.

Purseglove JW. 1975. Raffia palms. In Tropical Monocotyledons (Vols 1 and 2), ELBS, Longman (eds). Cambridge University Press: Cambridge; 439-440.

Robbrecht E. 1996. Geography of African Rubiaceae with reference to glacial rain forest refuges. In The Biodiversity of African Plants, van der Maesen LJG, van der Burgt XM, van Medenbach de Rooy JM (eds). Kluwer Academic Publishers: Dordrecht; 564-581.

Roux JP. 2009. Synopsis of the Lycodiophyta and Pteridophyta of Africa, Madagascar and Neighbouring Islands. South African National Biodiversity Institute: Pretoria Strelitzia.
Sosef MS. 1994. Refuge begonias: taxonomy, phylogeny and historical biogeography of Begonia sect. Loasibegonia and sect. Scutobegonia in relation to glacial rain forest refuges in Africa. Wageningen Agricultural University Papers, 94(1): 1306.

Stévart T. 2003. Étude taxonomique, écologique et phytogéographique des Orchidaceae en Afrique Centrale Atlantique. Thèse de Doctorat en Sciences Biologiques, Université libre de Bruxelles, Belgique, p.225.

White F. 1983. The vegetation of Africa. Descriptive memoirs to accompany the UNESCO, Association pour l'Etude Taxonomique de la Flore d'Afrique Tropicale.

White F. 1986. La Végétation de l'Afrique. Mémoire Accompagnant la Carte de Végétation de l'Afrique. UNESCO/ AETFAT/UNSO. ORSTOM et UNESCO: Paris.

White F. 1993. The chorological classification of history, methods and application. Bulletin du Jardin Botanique National de Belgique, 62: 225-282.

White L, Edwards A. 2001. Conservation en forêt pluviale africaine: Méthodes de Recherche. Wildlife Conservation Society: New York, U.S.A.

White L, Vande weghea JP. 2008. Patrimoine Mondial et Naturel d'Afrique centrale. Rapport de l'atelier de Brazzaville du 1214 mars. Unesco - CAWHFI. 